\title{
SOBRE PROCEDIMIENTOS DE FORMACION DE RELATIVAS EN ESPAÑOL ANTIGUO
}

Javier Elvira

0. Una de las construcciones que Gessner, en su clásico artículo sobre los pronombres relativo-interrogativos en español ${ }^{1}$, incluye bajo la ambigua rúbrica de que adverbial es aquella en que la función del relativo es señalada por un pronombre en la proposicián subordinada. La función señalada puede ser la de objeto directo o indirecto, así como la de régimen preposicional. Gessner cita ejemplos similares a los siguientes:

una fija que nunca mas la cuido veyer, / hela aqui fallada, debe a vos plazer (Apol.: 561cd)

las feridas primeras que las aya otorgadas (Cid: 1209) aquellos que gelas dieran non gelo avien logrado (ibid: 2425)

un baron a en to regno que espiritu de Dios es en el (Faz.: 178)

e en el tercero fabla en las questiones e en las cosas

que a omne menester en ellas (LConp. I, 1b: 40-42)

Es posible también encontrar casos en los que el elemento que retoma la función es un adverbio:

non dexo en regno casa que y non miso (SMillán, 460d)

Igualmente, la función de genitivo puede favorecer la aparicción de estas construcciones:

Ay otro arboles que la su fruta se come lo de fuera et non lo de dentro (LCE- XXXXIV: 45-46)

e sera omne que escucharan sus dichos (LConp. V, 211d: 7-8)

El giro no es extraño al latín, al menos en el registro popular, MeyerLubke, por su parte ${ }^{2}$, cita ejemplos similares de otras lenguas románicas, como la rumana o italiana.

1. "Das spaniche Relativ-und Interrogativpronomen", Zeitschrift für romanische Philologie, XVIII, 1894, pp. 449-497.

2. Grammaire des langues romanes, vol. III, n. ${ }^{\circ} 628$, Paris, 1900. 
El objetivo de este trabajo es proponer un análisis de las construcciones citadas que permita entrever las condiciones que han podido propiciar su aparición.

1. De los usos que hemos citado debemos separar, en primer lugar, aquellos casos en que el elemento que expresa la función de la subordinada es un pronombre átono de objeto directo o indirecto, de aquellos otros en que se trata de un pronombre regido por preposición o un adverbio. Los ejemplos del primer tipo deben encudrarse dentro de un fenómeno de alcance más general como es el de la redundancia pronominal. Marcos Marín ${ }^{3}$ estudia el pronombre pleonástico en proposiciones relativas, pero lo considera como una parcela dentro del más amplio terreno de la redundancia con pronombres átonos de objeto directo e indirecto. Sus conclusiones, de alcance igualmente general, permiten poner en relación el fenómeno con la tendencia a la distinción entre persona y no-persona, característica de la forma interior del español ${ }^{4}$; el estudio comparativo que efectua Marcos Marín en lenguas indoeuropeas, como las célticas, y no indoeuropeas, como el vasco y el árabe, nos informa tanto de la universalidad del fenómeno como de la posibilidad de que el contacto que con estas lenguas mantuvo el castellano pudiera ser un elemento coadyuvante en el desarrollo de la redundan$\mathrm{cia}^{5}$.

2. Que la redundancia pronominal tenga un alcance más general no obsta para que busquemos motivos de otra índole que puedan estar detrás de la aparición de estos usos, especialmente los de genitivo o adverbio, a los que no podemos aplicar la explicación anterior. Con tal fin nos parece oportuno recordar ciertos aspectos particulares de la evolución de la partícula que.

Es sabido que en romance que funcionó desde muy pronto como una suerte de relativo y/o conjunción universal, una especie de comodín gramatical del que la lengua se sirvió para la expresión de cualquier relación de subordinación ${ }^{6}$. El contexto o la colaboración del oyente permitían determinar en cada caso el tipo de subordinación que se expresaba. Para que se llegara a esta situación tuvieron que producirse dos hechos independientes: la ruina de las declinaciones casuales, por una parte, y la extensión del relativo a usos que en latín clásico estaban casi totalmente reservados a las conjunciones?.

3. Cf. Estudios sobre el pronombre, Madrid, Gredos, 1972, pp. 71-122.

4. Cf. R. Lapesa: "Evolución lingüística y forma interior del español" en Actas del XI Cong. Int. de Ling. y Fil. Rom., 1965, Madrid, CSIC, 1968.

5. H. S. Gehman ha querido ver en las construcciones relativas con pronombre redundante en el Cantar de Mio Cid y en El Quijote una influencia de la sintaxis árabe; cf. "Arabic Syntax of the relative pronoun in the $P$. del $C$ and D. Q." Hispanic Review, L, 1, pp. 53-60

6. Cf. R. Menéndez Pidal, Cantar de Mio Cid. Texto, gramática y vocabulario, $4^{\mathrm{a}}$., Madrid, Espasa-Calpe, 1969, pp. 813-814.

7. Cf. V. Väänänen, Introducción al latín vulgar, Madrid, Gredos, 1975, p. 254; E. Bourciez, Elements de linguistique romane, Paris, Klincksiek, 1967. 
Entre los diversos valores que la nueva conjunción adquiere se encuentra el consecutivo ${ }^{8}$, lo que permite a que en castellano antiguo y moderno funcionar como correlato de los pronombres tal y tanto. El valor consecutivo de que se detecta incluso en los casos en que los correlatos citados se encuentran ausentes. Bello ${ }^{9}$ opina que en la frase:

En lugar de una reverencia hizo una cabriola, que se levantó dos varas de medir en el aire (Cervantes)

hay que sobreentender la elipsis de tal antes de que. Por el contrario, según García de Diego ${ }^{10}$, hay que tener presente que diversos indefinidos pueden adquirir sentido comparativo y cita ejemplos como:

Hay cada montaña que asusta

Dicen unas cosas que avergüenzan

Si se concede, pues, la posibilidad de que el caracter indefinido del antecedente proporciona a que valor consecutivo, algunas de las frases como las citadas al principio de este trabajo pueden recibir una interpretación diferente. Compárense, por ejemplo, las dos siguientes:

mostró muchos milagros sobre esta labor, /que non podrié contarlos de mi mucho mejor (SMillán: 315cd)

por ende quer[r]ía que me contasedes tales cosas que las sopiesse yo (LCE-XXXVI: 64-65).

En ambos casos encontramos un pronombre átono de objeto directo que señala en la subordinada la función del antecedente (del relativo en un caso y de que consecutivo en el otro). En la frase de Berceo estaríamos inclinados a pensar que el enclítico -los es un pronombre redundante, mientras que no lo sería en la frase de Don Juan Manuel, donde el pronombre las desempeña una función reproductora imprescindible en una proposición consecutiva. Ahora bien, de acuerdo con lo dicho anteriormente, la partícula que de la primera frase podría interpretarse, como en la segunda, como una conjunción consecutiva en correlación con el indefinido muchos. El propio sentido de la frase parece favorecer este análisis, que excluye la consideración de -los como pronombre redundante.

Igualmente, la frase

Ay otros arboles que la su fruta se come lo de fuera et non lo de dentro (LCE-XXXXIV: 45-46)

8. La introducción de que en la correlación consecutiva es también un fenómeno latino vulgar. Väänänen (op. cit.) presenta el siguiente ejemplo de la Gesta Francorum: a tantilla gente Chistianorum, quod in pugillo potest claudi (G. F., 39, 116: "por un número tan reducido de cristianos que cabían en un puño")

9. Gramática de la lengua castellana, $7^{\mathrm{a}}$ ed., B. Aires, Sopena, 1964; véase también Narbona, A.: Las proposiciones consecutivas en español medieval, Granada, Univ., 1978, p. 138.

10. Gramática histórica española, $3^{\mathrm{a}}$ ed, Madrid, 1970, pp. 400-401. 
podría entenderse como

Ay otros árboles de cuya fruta se come lo de fuera...

o bien

Ay otros árboles tales que (de) la su fruta se come lo de fuera...

$\mathrm{El}$ análisis que hemos proporcionado nos ha sido sugerido por la interpretación que Bourciez efectúa de hechos similares del antiguo provenzal ${ }^{11}$. De acuerdo con este autor podemos distinguir los tres tipos básicos siguientes:

I. mostró muchos milagros sobre esta labor que non podría contar de mi mucho mejor

II. mostró tantos milagros sobre esta labor que non podría contarlos de mi mucho mejor

III. mostró muchos milagros sobre esta labor que non podría contarlos de mi mucho mejor.

Según Bourciez ${ }^{12}$, el cruce sintáctico entre relativas (I) y consecutivas (II) da lugar a lo que él llama relativo analítico (III), susceptible de recibir la doble interpretación sintáctica que hemos comentado. El alcance de esta hipótesis (si es correcta) es reducido, pues el valor consecutivo de las frases no se presenta claro en todos los casos; pero no debemos olvidar que el cruce sintáctico, si lo hubo, debió ser propiciado por la ampliación de los usos del antiguo relativo, que pasó a convertirse en muchos casos en un elemento puramente subordinador con usos muy variados.

3. Es probable que el estudio de estas construcciones tenga relevancia en la reciente discusión en torno a la naturaleza de las reglas sintácticas suscitada por aquellos que sostienen que tales reglas son "dependientes de la estructura" e insensibles a informaciones funcionales, frente a los que sostienen que las reglas sintácticas deben ser formuladas en términos de funciones gramaticales. En el terreno de la formación de relativas y desde una perspectiva funcional, Keenan y Comrie ${ }^{13}$, basándose en un extenso corpus interlingüístico, han sostenido que las lenguas varían en relación al número o tipo de posiciones sintácticas (funciones) relativizables y que tal variación viene gobernada por principio universal que regula la siguiente jerarquía:

$\mathrm{S}>$ OD $>$ OI $>$ OBL $>$ GEN $>$ TComp

(donde $\mathrm{S}=$ Sujeto; $\mathrm{OD}=$ Objeto directo; $\mathrm{OI}=$ Término de comparación; $>$ indica que la función sintáctica que se sitúa a su izquierda ocupa una posición de mayor jerarquía que la situada a su derecha).

11. "Note sur le relatif analitique en vieux-provençal" Revue des langues romanes, 67, 19331936, pp. 471-487.

12. Ibid., p. 481.

13. "Noun Phrase Accesibility and Universal Grammar", Linguistic Inquiry, VIII, I, 1977, pp. 65-99. 
Las bases en las que parece apoyarse esta jeraquía no son solamente los datos empíricos de diferentes lenguas sino también, por lo menos, otros hechos de caracter psicológico. Keenan y Comrie apoyan sus afirmaciones en estudios de carácter experimental efectuados en niños de diferentes edades que muestran que la capacidad de comprensión y el índice de error sintáctico en la construcción de subordinadas relativas se acomoda a la jerarquía de funciones proporcionada ${ }^{14}$.

Los datos de las lenguas examinadas por Keenan y Comrie permiten a estos enunciar el siguiente principio general: si una lengua manifiesta la posibilidad de relativizar una cierta posición en la jerarquía, siempre podráa relativar, por medio de la misma "estrategia" o de varias, todas las funciones que le preceden, aunque no necesariamente las que la siguen. Esto quiere decir que existen lenguas que pueden relativizar todas las posiciones, otras qu podrán relativizar GEN, OBL, OI, OD y S, pero no OComp, etc; como consecuencia de lo anterior, confirmada por los datos de Keenan y Comrie, la función de sujeto resulta ser universalmente relativizable.

Los tipos de estrategia susceptibles de ser utilizados por las lenguas son variados y su clasificación ha sido perfilada por otros autores ${ }^{15}$. Una de ellas es la llamada Retención del pronombre ("Pronoun Retention") ${ }^{16}$ que da lugar a construcciones similares a las que encabezaron este trabajo; en efecto, la mencionada estrategia consiste en la incorporación de un elemento pronominal en la subordinada que permita indentificar la función desempeñada en ésta por el antecedente. Este procedimiento de formación de relativas tiene la peculiaridad de que puede aplicarse a posiciones más "difíciles" (esto, es, más bajas en la jerarquía anterior); y son, por otro lado, más explícitas desde un punto de vista lógico ${ }^{17}$.

El procedimiento a que nos referimos y, por consiguiente, el tipo de estructura sintáctica a que da lugar, no constituyen una peculiaridad de lenguas aisladas. Keenan y Comrie las registran, entre otras, en las lenguas semíticas, chino, árabe, griego moderno, checo coloquial, persa, turco, etc.

Nótese que, salvo error por nuestra parte, el fenómeno de la retención del pronombre no se documenta en español cuando la función que se trata de señalar es la de sujeto. Este hecho confirma igualmente la tipología de Keenan y Comrie, que sólo encuentran una lengua (urhobo) ${ }^{18}$ en que tal circunstancia se produzca, frente a 24 que prescinden de la retención y utilizan para la relativización del sujeto otras estrategias menos explícitas.

14. Cf. K. y C., op. cit. p. 89.

15. Cf. D. Maxwell: "Strategies of relativización and NP accesibility"? Language, LV, 2, 1979, pp. 352-371.

16. Cf. Keenan y Comrie, op. cit. p. 92.

17. Cf. E. L. Keenan: "Logical expressive power and syntactic variation in natural language", en Keenan (ed): Formal Semantics of natural Language, Cambridge, C. Univ. Press, 1975.

18. K. y C. op. cit., p. 93. 
Cabe pensar que debajo de estos hechos, de carácter aparentemente universal, subyace una tendencia a la simplicidad sintáctica. Así lo ha visto Anna G. Ramat ${ }^{19}$, que considera que estas construcciones permite al relativo descargarse de una de las dos posibles funciones que suele desempeñar (la de marcar la función del antecedente en la subordinada), quedándose como un mero indicador de subordinación, al tiempo que permite a la frase relativa conservar un orden de palabras no marcado.

4. Por lo dicho en páginas anteriores, parece claro que son posibles interpretaciones diversas del tipo de construcciones que han suscitado este trabajo. Estas interpretaciones tienen diferente alcance pero no son incompatibles. Hemos hecho notar, por un lado, que la aparición del pronombre a veces llamado redundante pudo estar motivada por la extensión que el relativo experimenta, ya desde el propio latín, hacia usos cada vez más desvinculados de su primitivo valor, evolución ésta que lo convierte en una partícula con valores subordinantes muy variados; se ha planteado igualmente la posibilidad de que la ambivalencia funcional de que en muchos casos haya propiciado el cruce con otros usos de que más claramente conjuncionales. Por otra parte, y al margen de estas posibles interferencias, se ha hecho notar que las construcciones que comentamos constan como un tipo posible de subordinada relativa, registrado ampliamente en la tipología lingüística y especialmente adecuado, por su carácter explícito, para señalar funciones sintácticas menos asequibles según una jerarquía de funciones relativizables establecida con pretensiones de universalidad.

\section{Abreviaturas}

Apol. Libro de Apolonio. Ed. de Manuel Alvar. Fund. Juan March. Ed. Castalia; Madrid, 1976.

Cid. Cantar de Mio Cid. Ed. de Ramón Menéndez Pidal, C. de M. C. Texto, gramática y vocabulario, Espasa-Calpe, Madrid, 1969.

Faz. Fazienda de Ultramar. Ed. de M. Lazar, Salamanca: "Acta Salmanticensia", 1965.

LCE. Libro del Cauallero et del Escudero, del Infante Don Juan Manuel. Ed. de J. M. Blecua en Obras Completas, vol. I, Gredos, Madrid, 1983.

LConp. El libro conplido en los judicios de las estrellas, de Alfonso X. Ed. de G. Hilty, R. A. E., Madrid, 1964.

SMillan. Vida de San Millán de la Cogolla, de G. de Berceo. Ed. de B. Dutton, Thamesis Books, Londres, 1967.

19. "Explorations on syntactic change (Relative clauses formation strategies)" Papers from the 5th Intern. Cong. of Historical Linguistics, Amsterdam, Benjamins, 1981. 